\title{
Association of Diabetes Mellitus and Biochemical Knee Cartilage Composition Assessed by T2 Relaxation Time Measurements: Data from the Osteoarthritis Initiative
}

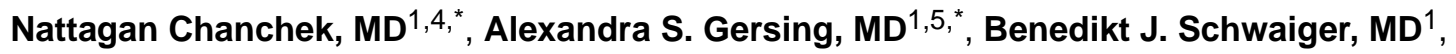 \\ Michael C. Nevitt, PhD², Jan Neumann, MD¹, Gabby B. Joseph, PhD1, Nancy E. Lane, MD $^{3}$, \\ Julia Zarnowski ${ }^{1}$, Felix C. Hofmann ${ }^{1}$, Ursula Heilmeier, MD' ${ }^{1}$, Charles E. McCulloch, PhD $^{2}$, \\ and Thomas M. Link, MD, PhD ${ }^{1}$ \\ ${ }^{1}$ Department of Radiology and Biomedical Imaging, University of California, 185 Berry Street, \\ Suite 350, San Francisco, CA 94107, USA 2Department of Epidemiology and Biostatistics, \\ University of California, San Francisco, Mission Hall, 550 16th St. 2nd Floor, San Francisco, CA \\ 94158, USA ${ }^{3}$ Department of Internal Medicine, UC Davis Medical Center, Sacramento, CA \\ 95817, USA ${ }^{4}$ Department of Radiology, Faculty of Medicine, Naresuan University, Phitsanulok, \\ Thailand ${ }^{5}$ Department of Radiology, Technical University of Munich, Ismaninger Strasse 22, \\ 81675 Munich, Germany
}

\section{Abstract}

Purpose-To investigate the association of presence and severity of diabetes mellitus (DM) with articular cartilage composition, using MRI-based T2 relaxation time measurements, and structural knee abnormalities.

\begin{abstract}
Materials and Methods-In the Osteoarthritis Initiative 208 participants with DM (age $63.0 \pm 8.9 \mathrm{y} ; 111$ females) and risk factors for osteoarthritis (OA) or mild radiographic tibiofemoral OA (Kellgren-Lawrence (KL) grade $\mathcal{S}$ ) were identified and group-matched with 208 controls without DM (age 63.3 $\pm 9.1 \mathrm{y} ; 111$ females). Subjects with diabetes-related renal or ophthalmological complications or insulin treatment at baseline $(\mathrm{n}=50)$ were defined as severe DM. 3T MR images of the right knee were assessed for articular cartilage T2, including texture and laminar analyses derived from the patella, medial and lateral femur and tibia and for structural abnormalities using the modified WORMS. Clustered linear regression analyses were used to assess associations of DM with MRI findings.
\end{abstract}

Results-DM subjects had significantly higher cartilage $\mathrm{T} 2$ in the patella (mean difference $0.92 \mathrm{~ms}$ [95\% confidence interval (CI) $0.79,1.06$ ]; $P=0.001$ ) and medial femur (mean difference $0.36 \mathrm{~ms}$ [95\% CI 0.27, 0.81]; $\mathrm{P}=0.006$ ) compared to controls. Averaged over all compartments DM subjects showed significantly higher texture parameters (variance, $P=0.001$; contrast, $P=0.002$; entropy, $P<0.001)$. Subjects with severe DM additionally showed higher T2 in the medial tibial

Corresponding author address: Alexandra S. Gersing, M.D., Department of Radiology and Biomedical Imaging, University of California, San Francisco, 185 Berry St, Suite 350, San Francisco, CA 94158, alexandra.gersing @ ucsf.edu.

*The first two authors contributed equally to this work. 
deep and superficial layers ( $P=0.011$ and $P=0.041)$ compared to controls. No significant differences in cartilage, meniscus and overall WORMS were found between the groups $(\mathrm{P}>0.05)$.

Conclusion-In comparison to non-diabetic controls, cartilage in DM subjects showed a higher and more heterogeneous cartilage $\mathrm{T} 2$ values, indicating increased articular cartilage degeneration. This affected even more compartments in subjects with severe DM.

\section{Keywords}

Knee osteoarthritis; diabetes; cartilage imaging; magnetic resonance imaging

\section{INTRODUCTION}

Diabetes mellitus (DM) is a metabolic disorder with an increasing prevalence in the last decade (1). The National Diabetes Statistics Report has estimated that approximately 29 million people in the US population have DM (2). Osteoarthritis (OA) causes a breakdown of cartilage and is associated with changes in adjacent knee joint structures such as soft tissue and subchondral bone that lead to structural knee joint abnormalities and consequently to clinical symptoms, such as pain and disability (3). OA and DM are frequently associated diseases, which may indicate that DM adversely affects articular cartilage and knee joint health and therefore may result in knee OA. On a cellular level, Type 2 DM typically demonstrates an insulin resistance. Consequently insulin activity is reduced resulting in prolonged hyperglycemia which leads to osmotic and oxidative stress and results in damage to the kidneys, eyes and other tissues (4). Previous studies have suggested that DM or hyperglycemia is a probable risk factor and prognostic predictor for OA (5-9). The Third National Health and Nutrition Examination Survey (NHANES III) has demonstrated that $11 \%$ of the patients with OA had DM, which was a significantly higher prevalence compared to the prevalence of $6 \%$ in the general population (10), suggesting DM is a risk factor for OA. Previous studies have also reported that hyperglycemia is significantly more prevalent in the population with OA versus the population without OA (30.7\% vs. $11.2 \%$, respectively) (11). High concentrations of fasting serum glucose, an indicator for hyperglycemia and therefore for DM, were also found to be associated with symptomatic $\mathrm{OA}$ in an epidemiological study (12), as well as adverse structural changes of knee joints with increased rates of tibial cartilage loss and incident bone marrow lesions $(12,13)$. DM and OA share many risk factors including aging, obesity, unhealthy dietary patterns, and physical inactivity. Moreover, the co-occurrence of DM and OA causes significantly more disability and significantly more pain in patients with OA compared to patients without OA (14). Even though studies investigating the association of DM and OA have been performed (4), the exact pathogenic role of DM in OA remains unclear.

Cartilage T2 relaxation time measurements reflect change of hydration and organization of anisotropic arrangement of collagen fibrils in the extracellular cartilage matrix (15). Knee cartilage T2 measurements have been used to assess cartilage degradation previously. Texture analysis using gray-level co-occurrence matrices (GLCM) has shown to allow early detection of compositional changes of cartilage in subjects with risk for OA, before radiographic evidence for $\mathrm{OA}$ is present by providing additional data on the $\mathrm{T} 2$ value distribution of neighboring pixels $(16,17)$. Laminar analysis, in which cartilage is separated 
into a deep bone and superficial articular layer, may also visualize early laminar disruption within cartilage when overall $\mathrm{T} 2$ does not yet reveal changes of cartilage composition (18).

The purpose of this study was to investigate the relationship between DM and cartilage composition to determine if an imaging biomarker could identify biochemical cartilage abnormalities in DM patients.

\section{METHODS}

\section{Subjects}

The Osteoarthritis Initiative is a longitudinal, multi-center cohort study that recruited 4796 participants prospectively from which subjects for this analysis were selected. Baseline age of the subjects included in this study ranged from 45 to 79 years and only subjects with symptomatic knee OA (progression cohort) or at risk for symptomatic knee OA (incidence cohort) were included. In this study, the re-release of the baseline clinical datasets (0.2.2) as well as the first release of the baseline imaging dataset of entire cohort group (0.E.1) were used.

We excluded subjects with moderate to advanced knee OA (Kellgren-Lawrence (KL) score 33) or missing KL score $(n=1104)$, since previous studies have shown that once advanced cartilage loss occurs, as found in individuals with higher KL score (19), T2 values may be limited for the evaluation of cartilage degradation (20). Moreover, subjects with a possible history of rheumatoid arthritis $(\mathrm{n}=206)$ and with missing clinical MR imaging or missing T2 mapping of the right knee at baseline were excluded from the analyses $(n=50)$. Using data from the Charlson comorbidity questionnaire developed by Katz et al. (21), self reported DM treated with either oral antidiabetic medication or insulin was present in 210 of the remaining subjects. Baseline body mass index (BMI) was not available in two of the DM subjects. Complete datasets were therefore available for 208 DM subjects. Subjects without DM were defined as subjects without self reported DM and without oral antidiabetic medication or insulin treatment $(n=3151)$. Of the subjects without $D M, 208$ subjects were randomly selected and group-matched to the previously selected subjects with DM. For a secondary analysis, DM subjects with severe disease were defined based on the Charlson comorbidity questionnaire data (21) as subjects with presence of diabetes-related renal or ophthalmological complications or insulin treatment (22) as assessed at baseline $(\mathrm{n}=50)$. The severe diabetes group is a subset of the diabetes group. The subject selection process is shown in Figure 1 and subject characteristics are presented in Table 1.

\section{MR imaging}

MR images of the right knee were obtained using four identical 3.0T scanners (Siemens Magnetom Trio; Siemens Healthcare, Erlangen, Germany) and quadrature transmit-receive coils (USA Instruments, Aurora, OH, USA) at four sites.

A sagittal 2D multi-slice multi-echo (MSME) spin-echo sequence with seven echo times $($ TEs $10 \mathrm{~ms}, 20 \mathrm{~ms}, 30 \mathrm{~ms}, 40 \mathrm{~ms}, 50 \mathrm{~ms}, 60 \mathrm{~ms}, 70 \mathrm{~ms}$; TR=2700 ms; field of view $(\mathrm{FOV})=$ $12 \mathrm{~cm}$, slice thickness $=3 \mathrm{~mm}$; gap $=0.5 \mathrm{~mm}$; in-plane spatial resolution $=0.31 \times 0.54 \mathrm{~mm}^{2}$ ) was used to obtain cartilage $\mathrm{T} 2$ relaxation times. The following four sequences were used 
for the morphological analysis of the cartilage: (i) a 2D intermediate-weighted (IW) turbo spin echo (TSE) sequence in the coronal plane $(3700 \mathrm{~ms} / 29 \mathrm{~ms}$, repetition time (TR)/ echo time (TE)); (ii) a 2D IW TSE sequences with fat suppression (FS) in the sagittal plane (3200ms/30ms, TR/TE); (iii) a 3D dual echo steady-state (DESS) gradient-echo with water excitation (WE) sequence obtained in the sagittal plane (16.3/4.7/25, $\mathrm{TR} / \mathrm{TE} / \mathrm{flip}$ angle), and (iv) a 3D T1-weighted fast low-angle shot (FLASH) gradient-echo with WE sequence in a coronal plane (20/7.57/12 ${ }^{\circ}$, TR/TE/flip angle). Further details regarding the MRI sequences analyzed can be found in the OAI protocol (23).

\section{MR Image analysis}

For T2 analysis, MR images of all subjects were analyzed by using an in-house, splinebased algorithm written in MATLAB (the Mathworks, Natick, Massachusetts), which was used for segmentation, as previously described $(24,25)$. The cartilage of five compartments (patella (PAT), medial femoral condyle (MF), lateral femoral condyle (LF), medial tibia (MT), and lateral tibia (LT)) was semi-automatically segmented by two trained researchers using the first echo of the sagittal 2D MSME sequence (Figure 2) and manually correcting the position of the points, in consensus and under supervision of an experienced radiologist. The trochlea (TRO) was not segmented due to flow artifacts caused by the popliteal artery. T2 values of each compartment were calculated by using a mono-exponential decay model as fitting function for the signal intensity using 6 echoes (TE 20-70 ms) after excluding the first echo in order to prevent possible errors due to the contribution of stimulated echoes to the overall MR signal in a multi-echo Carr-Purcell-Meiboom-Gill Sequence $(24,26)$ and using 3 parameter fittings accounting for noise $(26,27)$. Mean T2 values were computed for each cartilage compartment, and the global T2 value for the overall knee joint was calculated from the mean of all compartments.

Laminar analysis algorithms automatically subdivided the cartilage of each compartment into a superficial layer (articular surface) and a deep layer (bone interface) of equal thickness (18). In addition, cartilage GLCM texture analysis was performed to evaluate the spatial distribution of cartilage $\mathrm{T} 2$ values within each cartilage compartment, reflecting heterogeneity of $\mathrm{T} 2$ values throughout the cartilage matrix, as a measure for cartilage degeneration (16,17,28-30). Based on our previous work, three GLCM texture parameters were included in the analysis: contrast (contrast group), entropy (orderliness group) and variance (statistics group) $(16,30)$. These additional analyses were performed and interpreted as described previously (30).

Morphological MR sequences from both groups were reviewed on a picture archiving communication system (PACS) workstations (Agfa, Ridgefield Park, NJ, USA) by two radiologists (N.C. and A.S.G. with 6 and 5 years of experience, respectively), blinded to patient information, using the semi-quantitative modified WORMS grading system, as previously described $(31,32)$. In cases of disagreement, a consensus reading was performed with a third more experienced musculoskeletal radiologist (T.M.L. with 23 years of experience).

Meniscal lesions were assessed in six regions (anterior/body/posterior regions of the medial and lateral menisci $)$ and graded from 0 to $4(0=$ normal, $1=$ intrasubstance abnormality, $2=$ 
non-displaced tear, $3=$ displaced or complex tear, and $4=$ complete destruction or maceration of the meniscus).

Cartilage lesions were evaluated in 6 regions (PAT, TRO, MF, LF, MT and LT) with an 8point scale: $0=$ normal, $1=$ normal thickness but increased or otherwise abnormal signal on fluid sensitive sequences, $2=$ partial-thickness focal defect $<1 \mathrm{~cm}$ in greatest width, $2.5=$ full-thickness focal defect $<1 \mathrm{~cm}$ in greatest width, $3=$ multiple areas of partial-thickness defects (grade 2) intermixed with areas of normal thickness, or grade 2 defect wider than 1 $\mathrm{cm}$ but $<75 \%$ of the entire region, $4=$ diffuse ( $275 \%$ of the region) partial-thickness loss, 5 $=$ multiple areas of full-thickness defect (grade 2.5 ) but $<75 \%$ of the region, and $6=$ diffuse ( $\geq 75 \%$ of the region) full-thickness loss.

Bone marrow edema pattern (BMEP) lesions were identified as poorly marginated areas of increased signal intensity in the normally fatty marrow on T2-weighted TSE images with FS and were graded in the subchondral zone of the same 6 regions as described in the cartilage score, using a scale from 0 to 3 based on lesion size: $0=$ none, $1=\operatorname{minimal}(<5 \mathrm{~mm}$ in diameter), $2=$ moderate $(5-20 \mathrm{~mm}$ in diameter $)$ and $3=$ severe $(>20 \mathrm{~mm}$ in diameter $)$.

Ligamentous abnormalities of the anterior cruciate ligament, posterior cruciate ligament, medial collateral ligament, lateral collateral ligament, patellar tendon, and popliteal tendon as well as other findings (subchondral cysts, effusion, loose bodies, and popliteal cysts) were graded according to WORMS as previously described (31). For each subscale a sum score was calculated by adding the lesions scores of all subregions of each knee and an overall WORMS score was estimated by adding all of these.

\section{Reproducibility}

To calculate both, the intra- and inter-reader reproducibility, the reproducibility error was assessed by calculating the root mean square average of the single coefficients of variation (CV) on a percentage basis, as previously reported (33). Inter-reader reproducibility was assessed in 10 randomly selected subjects between the two readers overall and for each of the five compartments segmented (PAT, MF, LF, MT, and LT). Averaged over all compartments, the inter-reader reproducibility for image segmentation for T2 evaluation was $1.93 \%$. The CVs for each compartment were $2.26 \%$ for PAT, $1.63 \%$ for MF, $1.59 \%$ for LF, $2.36 \%$ for MT, and $1.83 \%$ for LT. For intra-reader reproducibility, both readers repeated the image segmentation for $\mathrm{T} 2$ evaluation in the same 10 randomly selected subjects with at least 14 days separating the readings. The intra-reader reproducibility for image segmentation over all compartments for $\mathrm{T} 2$ evaluation was $1.12 \%$ and $2.06 \%$, respectively. Intra-reader CVs were calculated for each compartment: $1.12 \%$ and $1.78 \%$ for PAT, $0.75 \%$ and $1.00 \%$ for MF, $0.64 \%$ and $1.63 \%$ for LF, $1.92 \%$ and $2.85 \%$ for MT, $1.18 \%$ and $2.80 \%$ for LT, respectively.

In order to calculate the intra- and inter-reader reproducibility of the WORMS grading, each of the two radiologists performed WORMS grading twice independently for 10 randomly selected subjects, the two readings of each reader were at least 14 days apart. Intra-class correlation coefficients (ICCs) were calculated in order to compare the WORMS overall and to compare each WORMS subscore (meniscus and cartilage) separately. The intra-reader 
agreement for overall WORMS grading were 0.85 (0.74-0.92) and $0.86(0.76-0.95), 0.85$

$(0.79-0.93)$ and $0.87(0.81-0.94)$ for meniscus WORMS and $0.87(0.81-0.92)$ and 0.84

$(0.78-0.95)$ for cartilage WORMS, respectively. ICCs for inter-reader agreement were 0.83

(0.74-0.95) for overall WORMS, $0.83(0.76-0.91)$ for meniscus WORMS and $0.80(0.74-$

0.87 ) for cartilage WORMS. Similar intra-reader and inter-reader agreements of WORMS gradings by our group have been published in previous studies $(25,34)$.

\section{Statistical Analysis}

The statistical analysis was performed with Stata Version 13 (StataCorp, College Station, TX) using a 2-sided 0.05 level of significance. T tests and Chi-square tests were used to assess the differences in continuous variables (age, BMI, height and Physical Activity Score for the Elderly (PASE)) and categorical variables (gender, KL grade of the right knee at baseline, race, health status, risk factors for osteoarthritis), respectively, between subjects with DM and controls without DM.

The differences between outcome variables (mean T2, laminar parameters, texture parameters, WORMS scores) between subjects with and without DM were assessed using clustered linear regression analyses adjusting for race and cluster pair. Similar analyses were performed to determine differences in outcome parameters between subjects with severe DM and controls without DM.

Due to a large number of outcome parameters, analyses were split into the following categories based on previously published analyses $(16,28,35,36)$ : primary outcomes (compartments: overall compartments, MT, PAT; imaging parameter: mean T2, deep layer T2 (laminar analysis), texture parameters contrast and variance); secondary outcomes (compartments: LF, LT and MF; imaging parameters: texture parameter entropy, overall WORMS, subscores cartilage and meniscus).

\section{RESULTS}

\section{Subject Characteristics}

Subject characteristics are illustrated in Table 1. The subjects with DM ( $\mathrm{n}=208$; mean age, $63.0 \pm 8.9$ years) were matched to 208 subjects from the control group without DM (mean age, $63.3 \pm 9.1$ years). Mean baseline BMI (mean \pm SD) of DM subjects and subjects without DM was similar $\left(31.0 \pm 4.4 \mathrm{~kg} / \mathrm{m}^{2}\right.$ and $31.2 \pm 4.5 \mathrm{~kg} / \mathrm{m}^{2}$, respectively; $(P=0.70)$ ). The subjects in the two groups showed no significant differences in age, height, the distribution of sex and KL scores, risk factors for osteoarthritis and health status $(P>0.05)$. In both groups, $65 \%$ of the subjects had either no signs $(\mathrm{KL}=0)$ or only doubtful signs of $\mathrm{OA}$ $(\mathrm{KL}=1)$. There were significant differences between the groups regarding the distribution of race $(P<0.001)$, therefore this variable was included into the regression analyses. Mean age of the severe DM subjects in this study $(\mathrm{n}=50)$ was $57.71 \pm 8.95$ years and BMI was 30.95 $\pm 4.26 \mathrm{~kg} / \mathrm{m}^{2}$. 


\section{T2 measurements}

Mean T2 values in subjects with and without DM are shown in Table 2. In the primary analyses, cartilage T2 measurements of the patella (mean difference $0.92 \mathrm{~ms}[95 \%$ confidence interval (CI) $0.79,1.06$ ]; $P=0.001$; Figure 3 ) and in the medial femur condyle (mean difference $0.36 \mathrm{~ms}$ [95\% confidence interval (CI) 0.27, 0.81]; $P=0.006$ ) were significantly elevated in subjects with DM compared to those without DM. In the laminar subanalyses, $\mathrm{T} 2$ of the deep layer was significantly elevated in the patella in the group with DM compared to the group without DM $(P<0.001)$. Moreover, the texture parameters GLCM variance, contrast and entropy showed significantly higher values in the global knee cartilage ( $P=0.001, P=0.002$ and $P<0.001$; Table 3 ), in the patella and medial tibia (for each, $P<0.01$; Figure 4$)$.

Mean T2 values in each compartment as well as averaged over all compartments were elevated in the group of severe DM subjects compared to the controls without DM. The T2 values of the individuals with severe DM were significantly elevated compared to the control group without DM in the patella as well as the medial femur condyle ( $P=0.002$ and $P=0.012$, respectively; Table 2) and reached an even higher level of significance in the cartilage T2 of the deep layer in the patella (mean difference $1.44 \mathrm{~ms}$ [95\% CI 1.02, 1.85], $P<0.001$ ).

Subjects with more severe DM also showed significantly elevated T2 values in the deep and superficial layer of medial tibia $(P=0.011$ and $P=0.041)$.

\section{Morphological knee abnormalities}

The prevalence of cartilage WORMS subscores of cartilage lesions was low in both groups in all compartments and did not differ significantly ( $P>0.05$; Table 4$)$, indicating a low degree of focal cartilage abnormalities and comparable morphological cartilage status between the subjects with and without DM. Moreover, there were no significant differences in the overall WORMS score and the meniscus WORMS subscores between the subjects with and without DM $(P>0.05)$. For subgroup analyses, subjects with severe DM showed no significant differences in the WORMS subscores in comparison to subjects with DM $(P>0.05)$.

\section{DISCUSSION}

In this study, the association between DM and the biochemical composition and texture of cartilage was assessed in individuals with risk factors for OA and mild OA, using 3T MRbased cartilage T2 relaxation time mapping. Subjects with DM showed significantly increased cartilage $\mathrm{T} 2$ values in the patella and medial femur compared to controls, indicating more advanced biochemical cartilage degradation. These findings are supported by the texture parameters GLCM variance, entropy and contrast in the patella and medial tibia as well as averaged over all compartments, showing significantly elevated texture parameters in individuals with DM compared to the controls without DM. Interestingly significant differences were found in even more compartments when comparing severe DM subjects to the controls without DM, showing significantly elevated cartilage T2 additionally in the deep and superficial layer of the medial tibia in individuals with severe DM compared to those without DM. These findings may suggest even more extensive cartilage 
degeneration in subjects with severe DM. Interestingly, however, we did not see significant differences in knee morphological abnormalities using the WORMS grading, suggesting that findings in DM patients predominantly affect the cartilage matrix.

The associations found between DM and elevated cartilage T2 values suggest that DM may have an effect on cartilage degeneration. DM is a modifiable metabolic disorder in which elevated blood sugar levels are present over a prolonged period of time. There are several studies revealing that DM may adversely affect the homeostasis and reparation of articular cartilage through different pathways. Previous studies have demonstrated that DM may favor the development or progression of OA (5-9). For example, when chondrocytes are exposed to high glucose levels over a longer period of time, they are unable to down regulate GLUT-1 protein, and therefore accumulate glucose and produce more reactive oxygen species (ROS) (37). An intracellular increase of glucose and ROS are major mediators for cartilage destruction which can promote cartilage degeneration (38). High glucose levels in vitro can impair the synthesis of type II collagen due to a diminished transportation of dehydroascorbate into chondrocytes, which overtime could reduce collagen quality (39). Also, the signal transmitted through the receptors for advanced glycation endproducts on chondrocytes leads to an overexpression of pro-inflammatory and pro-degradative mediators and therefore can adversely affect chondrocytes. Moreover, increased glucose and sorbitol levels cause osmotic stress mechanisms, accelerating fibrocartilage matrix catabolism in the intervertebral discs of patients with DM (40). Furthermore, insulin-like growth factor-1 (IGF-1) and inflammation may have a role for DM and OA acceleration (41).

In order to investigate potential cartilage impairment and its association with DM, 3T MRIbased T2 relaxation time mapping was used. Cartilage T2 mapping detects an increase in water content and disruption of the organization of the anisotropic arrangement of collagen fibrils in the extracellular cartilage matrix (15), visualized as cartilage T2 relaxation time increase (42) and is therefore a useful tool to estimate early cartilage degeneration before irreversible cartilage loss occurs. Since studies had suggested that metabolic risk factors, including obesity, DM, hypertension and dyslipidemia are significantly associated to both occurrence and progression of knee OA (43), a previous study found that certain metabolic risk factors (high abdominal circumference, hypertension, high fat consumption and diabetes) were associated with elevated baseline T2 values (35). In the latter study the only results remaining statistically significant after adjusting for baseline BMI were the associations with diabetes. Therefore, in this study we primarily focused on DM by using a larger cohort with DM and by isolating the effects of DM on OA through our statistical analyses, including cartilage texture analysis. In the texture analysis cartilage GLCM texture values averaged over all compartments as well as in each assessed compartment was elevated in individuals with DM and with severe DM compared to those without DM, indicating more severe cartilage degeneration in patients with DM averaged over all compartments compared to controls without DM. These findings are in line with those of previous studies showing that quantitative analysis of cartilage GLCM texture parameters variance, contrast and entropy allows earlier detection of biochemical changes within the cartilage before morphological evidence for OA has occured, by providing information on the spatial distribution of $\mathrm{T} 2$ pixel values (16). 
Moreover, there have been associations reported between hand OA and metabolic syndrome components, showing a strong correlation of OA with DM (44). The findings of our secondary analysis that focused on patients with severe DM revealed significantly elevated cartilage $\mathrm{T} 2$ in the deep layer of the cartilage of the patella and additionally in the medial tibia compared to non-diabetic controls, suggesting that there may be an association between not only presence, but also severity of DM and cartilage degeneration.

Cartilage T2 has been shown to correlate with the severity of morphological degenerative change in the cartilage and meniscus (24). It should be noted that for the selection process of our study cohort we were limited to the KL grade for the assessment of the OA status of the subjects. Yet, after performing an analysis of the degenerative morphological findings, the cartilage lesion score appeared to be very low over all subjects analyzed and there were no significant differences found between the group with and without DM, neither regarding cartilage lesions nor regarding any other morphological abnormality assessed. Therefore, cartilage $\mathrm{T} 2$ differences between the groups were not caused by differences of the severity of morphological degenerative abnormalities between the groups.

Nevertheless, this study has several limitations. Firstly, this work is a cross-sectional observational study. Therefore, we cannot establish whether cartilage changes occurred before or after development of diabetes. And, there are confounding factors, which may affect the association between diabetes status and T2 values. Although our matching system allowed us to control for certain OA-related confounders, we were not able to control for all factors linked to OA, for example smoking, treatment for OA, and other comorbidities. Previous studies have reported that the collagen content and its orientation is the major factor in changes of cartilage $\mathrm{T} 2$ relaxation times (45-48). Biochemical analyses of the cartilage compositional changes were not able to be performed in this study. Based on previous studies, $\mathrm{T} 2$ relaxation time imaging in individuals with diabetes may suggest increased articular cartilage degeneration compared to individuals without diabetes, yet future studies with biochemical analyses are needed in order to confirm these assumptions histologically. Moreover, plasma glucose or hemoglobin A1c levels were not available to determine associations of biochemical cartilage composition with degree of glycemic control. Longitudinal studies may provide further insight on the effect of DM on OA progression.

In conclusion, elevated $\mathrm{T} 2$ values and texture parameters in DM subjects compared to controls may indicate altered biochemical composition possibly associated with cartilage degeneration, while there were no significant differences found in morphological knee joint abnormalities. Associations of DM with elevated T2 values were observed in even more compartments when comparing subjects with severe DM and controls without DM, which may suggest an association of more advanced biochemical cartilage degradation with severe DM.

\title{
Acknowledgments
}

\author{
Grant Support
}


The OAI is a public-private partnership comprised of five contracts (N01-AR-2-2258; N01-AR-2-2259; N01AR-2-2260; N01-AR-2-2261; N01-AR-2-2262) funded by the National Institutes of Health, a branch of the Department of Health and Human Services, and conducted by the OAI Study Investigators. Private funding partners include Pfizer, Inc.; Novartis Pharmaceuticals Corporation; Merck Research Laboratories; and GlaxoSmithKline. Private sector funding for the OAI is managed by the Foundation for the National Institutes of Health. This manuscript has received the approval of the OAI Publications Committee based on a review of its scientific content and data interpretation. The analyses in this study were funded through the NIH (National Institute of Arthritis and Musculoskeletal and Skin Diseases grants R01AR064771 and P50-AR060752). This manuscript has received the approval of the OAI Publications Committee based on a review of its scientific content and data interpretation.

\section{References}

1. Guariguata L, Whiting DR, Hambleton I, Beagley J, Linnenkamp U, Shaw JE. Global estimates of diabetes prevalence for 2013 and projections for 2035. Diabetes Res Clin Pract. 2014; 103(2):137149. [PubMed: 24630390]

2. National Diabetes Statistics Report. Estimates of Diabetes and Its Burden in the United States. 2014.

3. Bijlsma JW, Berenbaum F, Lafeber FP. Osteoarthritis: an update with relevance for clinical practice. Lancet. 2011; 377(9783):2115-2126. [PubMed: 21684382]

4. King KB, Rosenthal AK. The adverse effects of diabetes on osteoarthritis: update on clinical evidence and molecular mechanisms. Osteoarthritis Cartilage. 2015; 23(6):841-850. [PubMed: 25837996]

5. Jingsheng S, Yibing W, Jun X, et al. MicroRNAs are potential prognostic and therapeutic targets in diabetic osteoarthritis. J Bone Miner Metab. 2015; 33(1):1-8. [PubMed: 25245120]

6. Kirkman MS. Osteoarthritis progression: is diabetes a culprit? Osteoarthritis Cartilage. 2015; 23(6): 839-840. [PubMed: 25827972]

7. Kim D, Song J, Ahn C, Kang Y, Chun CH, Jin EJ. Peroxisomal dysfunction is associated with upregulation of apoptotic cell death via miR-223 induction in knee osteoarthritis patients with type 2 diabetes mellitus. Bone. 2014; 64:124-131. [PubMed: 24727161]

8. Eymard F, Parsons C, Edwards MH, et al. Diabetes is a risk factor for knee osteoarthritis progression. Osteoarthritis Cartilage. 2015; 23(6):851-859. [PubMed: 25655678]

9. Schett G, Kleyer A, Perricone C, et al. Diabetes is an independent predictor for severe osteoarthritis: results from a longitudinal cohort study. Diabetes Care. 2013; 36(2):403-409. [PubMed: 23002084]

10. Singh G, Miller JD, Lee FH, Pettitt D, Russell MW. Prevalence of cardiovascular disease risk factors among US adults with self-reported osteoarthritis: data from the Third National Health and Nutrition Examination Survey. Am J Manag Care. 2002; 8(15 Suppl):S383-391. [PubMed: 12416788]

11. Puenpatom RA, Victor TW. Increased prevalence of metabolic syndrome in individuals with osteoarthritis: an analysis of NHANES III data. Postgrad Med. 2009; 121(6):9-20. [PubMed: 19940413]

12. Cimmino MA, Cutolo M. Plasma glucose concentration in symptomatic osteoarthritis: a clinical and epidemiological survey. Clin Exp Rheumatol. 1990; 8(3):251-257. [PubMed: 2379341]

13. Davies-Tuck ML, Wang Y, Wluka AE, et al. Increased fasting serum glucose concentration is associated with adverse knee structural changes in adults with no knee symptoms and diabetes. Maturitas. 2012; 72(4):373-378. [PubMed: 22739094]

14. Reeuwijk KG, de Rooij M, van Dijk GM, Veenhof C, Steultjens MP, Dekker J. Osteoarthritis of the hip or knee: which coexisting disorders are disabling? Clin Rheumatol. 2010; 29(7):739-747. [PubMed: 20177725]

15. Liess C, Lusse S, Karger N, Heller M, Gluer CC. Detection of changes in cartilage water content using MRI T2-mapping in vivo. Osteoarthritis Cartilage. 2002; 10(12):907-913. [PubMed: 12464550]

16. Joseph GB, Baum T, Carballido-Gamio J, et al. Texture analysis of cartilage T2 maps: individuals with risk factors for OA have higher and more heterogeneous knee cartilage MR T2 compared to normal controls--data from the osteoarthritis initiative. Arthritis Res Ther. 2011; 13(5):R153. [PubMed: 21933394] 
17. Mosher TJ, Dardzinski BJ, Smith MB. Human articular cartilage: influence of aging and early symptomatic degeneration on the spatial variation of T2--preliminary findings at $3 \mathrm{~T}$. Radiology. 2000; 214(1):259-266. [PubMed: 10644134]

18. Carballido-Gamio J, Blumenkrantz G, Lynch JA, Link TM, Majumdar S. Longitudinal analysis of MRI T(2) knee cartilage laminar organization in a subset of patients from the osteoarthritis initiative. Magn Reson Med. 2010; 63(2):465-472. [PubMed: 19918905]

19. Guermazi A, Eckstein F, Hayashi D, et al. Baseline radiographic osteoarthritis and semiquantitatively assessed meniscal damage and extrusion and cartilage damage on MRI is related to quantitatively defined cartilage thickness loss in knee osteoarthritis: the Multicenter Osteoarthritis Study. Osteoarthritis Cartilage. 2015; 23(12):2191-2198. [PubMed: 26162806]

20. Jungmann PM, Kraus MS, Nardo L, et al. T(2) relaxation time measurements are limited in monitoring progression, once advanced cartilage defects at the knee occur: longitudinal data from the osteoarthritis initiative. J Magn Reson Imaging. 2013; 38(6):1415-1424. [PubMed: 24038491]

21. Katz JN, Chang LC, Sangha O, Fossel AH, Bates DW. Can comorbidity be measured by questionnaire rather than medical record review? Medical care. 1996; 34(1):73-84. [PubMed: 8551813]

22. American Diabetes A. Diagnosis and classification of diabetes mellitus. Diabetes care. 2014; 37(Suppl 1):S81-90. [PubMed: 24357215]

23. Peterfy CG, Schneider E, Nevitt M. The osteoarthritis initiative: report on the design rationale for the magnetic resonance imaging protocol for the knee. Osteoarthritis and cartilage / OARS, Osteoarthritis Research Society. 2008; 16(12):1433-1441.

24. Joseph GB, Baum T, Alizai H, et al. Baseline mean and heterogeneity of MR cartilage T2 are associated with morphologic degeneration of cartilage, meniscus, and bone marrow over 3 years-data from the Osteoarthritis Initiative. Osteoarthritis Cartilage. 2012; 20(7):727-735. [PubMed: 22503812]

25. Baum T, Stehling C, Joseph GB, et al. Changes in knee cartilage T2 values over 24 months in subjects with and without risk factors for knee osteoarthritis and their association with focal knee lesions at baseline: data from the osteoarthritis initiative. J Magn Reson Imaging. 2012; 35(2):370378. [PubMed: 21987496]

26. Raya JG, Dietrich O, Horng A, Weber J, Reiser MF, Glaser C. T2 measurement in articular cartilage: impact of the fitting method on accuracy and precision at low SNR. Magn Reson Med. 2010; 63(1):181-193. [PubMed: 19859960]

27. Miller AJ, Joseph PM. The use of power images to perform quantitative analysis on low SNR MR images. Magn Reson Imaging. 1993; 11(7):1051-1056. [PubMed: 8231670]

28. Carballido-Gamio J, Joseph GB, Lynch JA, Link TM, Majumdar S. Longitudinal analysis of MRI T2 knee cartilage laminar organization in a subset of patients from the osteoarthritis initiative: a texture approach. Magn Reson Med. 2011; 65(4):1184-1194. [PubMed: 21413082]

29. Haralick R, Shanmugam K, Dinstein I. Textural Features for Image Classification. IEEE Transactions on Systems, Man, and Cybernetics. 1973; SMC-1:610-618.

30. Gersing AS, Solka M, Joseph GB, et al. Progression of cartilage degeneration and clinical symptoms in obese and overweight individuals is dependent on the amount of weight loss: 48month data from the Osteoarthritis Initiative. Osteoarthritis and cartilage / OARS, Osteoarthritis Research Society. 2016; 24(7):1126-1134.

31. Peterfy CG, Guermazi A, Zaim S, et al. Whole-Organ Magnetic Resonance Imaging Score (WORMS) of the knee in osteoarthritis. Osteoarthritis Cartilage. 2004; 12(3):177-190. [PubMed: 14972335]

32. Baum T, Joseph GB, Arulanandan A, et al. Association of magnetic resonance imaging-based knee cartilage T2 measurements and focal knee lesions with knee pain: data from the Osteoarthritis Initiative. Arthritis Care Res (Hoboken). 2012; 64(2):248-255. [PubMed: 22012846]

33. Gluer CC, Blake G, Lu Y, Blunt BA, Jergas M, Genant HK. Accurate assessment of precision errors: how to measure the reproducibility of bone densitometry techniques. Osteoporosis international: a journal established as result of cooperation between the European Foundation for Osteoporosis and the National Osteoporosis Foundation of the USA. 1995; 5(4):262-270. 
34. Pan J, Pialat JB, Joseph T, et al. Knee cartilage T2 characteristics and evolution in relation to morphologic abnormalities detected at 3-T MR imaging: a longitudinal study of the normal control cohort from the Osteoarthritis Initiative. Radiology. 2011; 261(2):507-515. [PubMed: 21900614]

35. Jungmann PM, Kraus MS, Alizai H, et al. Association of metabolic risk factors with cartilage degradation assessed by $\mathrm{T} 2$ relaxation time at the knee: data from the osteoarthritis initiative. Arthritis Care Res (Hoboken). 2013; 65(12):1942-1950. [PubMed: 23926027]

36. Serebrakian AT, Poulos T, Liebl H, et al. Weight loss over 48 months is associated with reduced progression of cartilage T2 relaxation time values: data from the osteoarthritis initiative. J Magn Reson Imaging. 2015; 41(5):1272-1280. [PubMed: 24700497]

37. Rosa SC, Goncalves J, Judas F, Mobasheri A, Lopes C, Mendes AF. Impaired glucose transporter-1 degradation and increased glucose transport and oxidative stress in response to high glucose in chondrocytes from osteoarthritic versus normal human cartilage. Arthritis Res Ther. 2009; 11(3):R80. [PubMed: 19490621]

38. Henrotin YE, Bruckner P, Pujol JP. The role of reactive oxygen species in homeostasis and degradation of cartilage. Osteoarthritis Cartilage. 2003; 11(10):747-755. [PubMed: 13129694]

39. McNulty AL, Stabler TV, Vail TP, McDaniel GE, Kraus VB. Dehydroascorbate transport in human chondrocytes is regulated by hypoxia and is a physiologically relevant source of ascorbic acid in the joint. Arthritis Rheum. 2005; 52(9):2676-2685. [PubMed: 16142743]

40. Cheng X, Ni B, Zhang Z, et al. Polyol pathway mediates enhanced degradation of extracellular matrix via 338 MAPK activation in intervertebral disc of diabetic rats. Connect Tissue Res. 2013; 54(2):118-122. [PubMed: 23215968]

41. Shoelson SE, Lee J, Goldfine AB. Inflammation and insulin resistance. J Clin Invest. 2006; 116(7): 1793-1801. [PubMed: 16823477]

42. Dunn TC, Lu Y, Jin H, Ries MD, Majumdar S. T2 relaxation time of cartilage at MR imaging: comparison with severity of knee osteoarthritis. Radiology. 2004; 232(2):592-598. [PubMed: 15215540]

43. Yoshimura N, Muraki S, Oka H, et al. Accumulation of metabolic risk factors such as overweight, hypertension, dyslipidaemia, and impaired glucose tolerance raises the risk of occurrence and progression of knee osteoarthritis: a 3-year follow-up of the ROAD study. Osteoarthritis Cartilage. 2012; 20(11):1217-1226. [PubMed: 22796312]

44. Dahaghin S, Bierma-Zeinstra SM, Koes BW, Hazes JM, Pols HA. Do metabolic factors add to the effect of overweight on hand osteoarthritis? The Rotterdam Study Ann Rheum Dis. 2007; 66(7): 916-920. [PubMed: 17314121]

45. Menezes NM, Gray ML, Hartke JR, Burstein D. T2 and T1rho MRI in articular cartilage systems. Magn Reson Med. 2004; 51(3):503-509. [PubMed: 15004791]

46. Mosher TJ, Dardzinski BJ. Cartilage MRI T2 relaxation time mapping: overview and applications. Semin Musculoskelet Radiol. 2004; 8(4):355-368. [PubMed: 15643574]

47. Nieminen MT, Rieppo J, Toyras J, et al. T2 relaxation reveals spatial collagen architecture in articular cartilage: a comparative quantitative MRI and polarized light microscopic study. Magn Reson Med. 2001; 46(3):487-493. [PubMed: 11550240]

48. Xia Y, Moody JB, Alhadlaq H. Orientational dependence of T2 relaxation in articular cartilage: A microscopic MRI (microMRI) study. Magn Reson Med. 2002; 48(3):460-469. [PubMed: 12210910] 


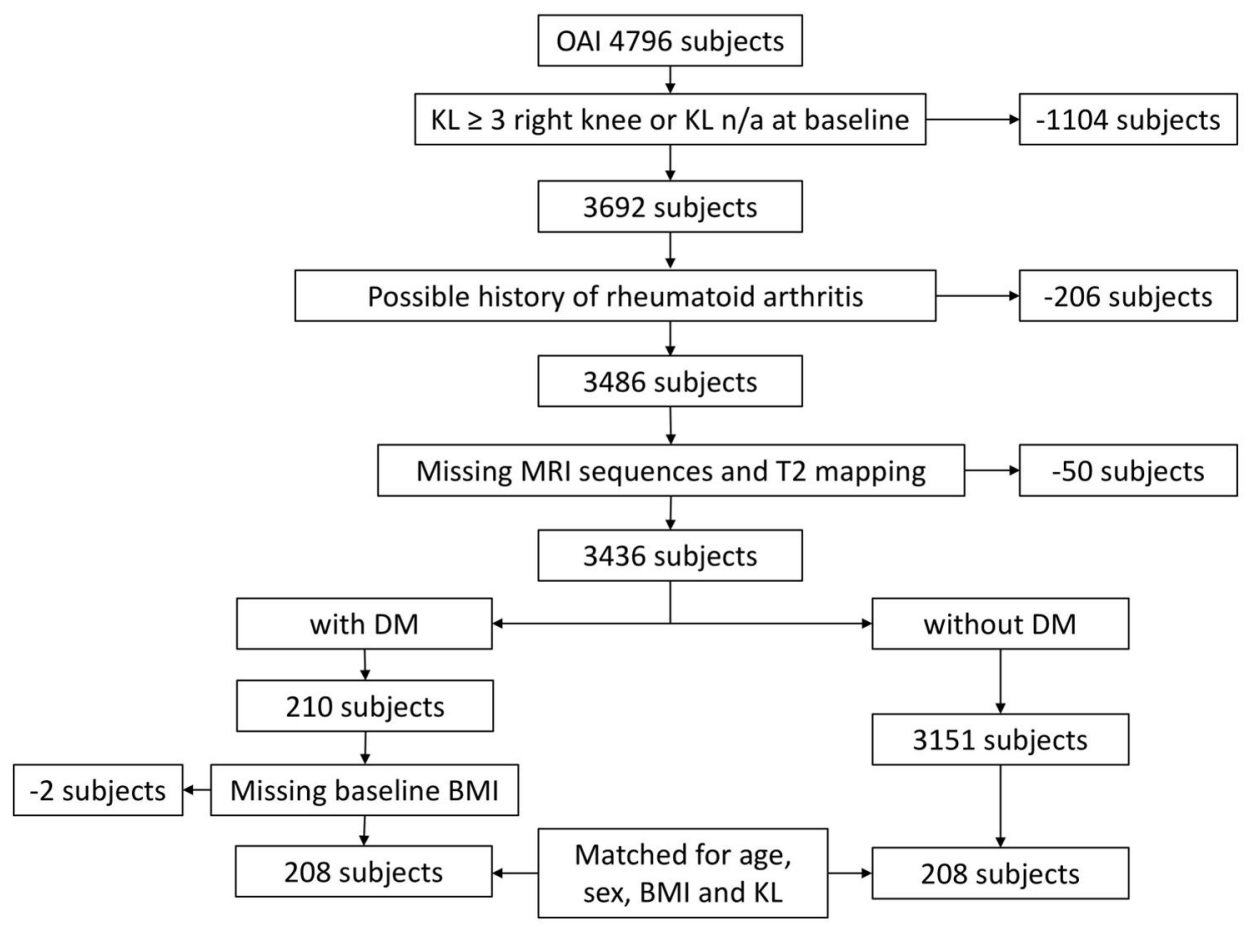

Figure 1.

Flowchart illustrating subject selection from OAI database 


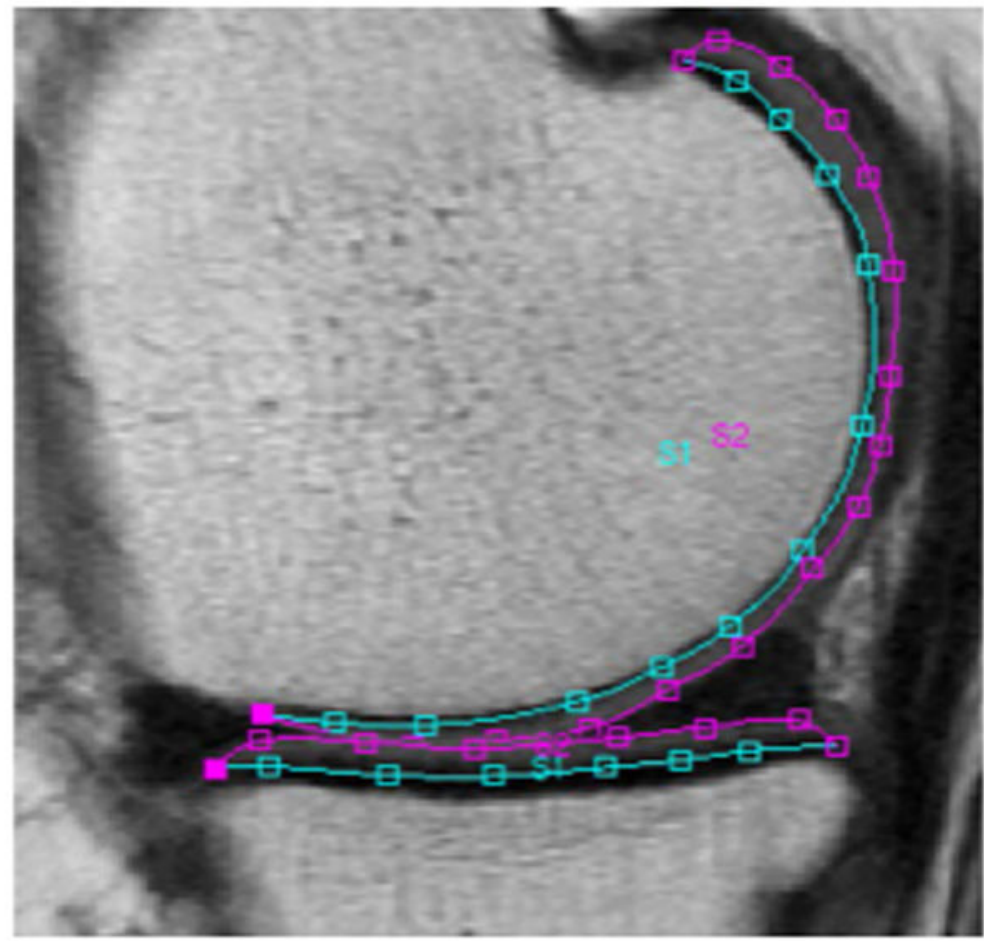

Figure 2.

Example of T2 segmentation splines of the medial compartment (medial femur and medial tibia) of the right knee of a study subject. 


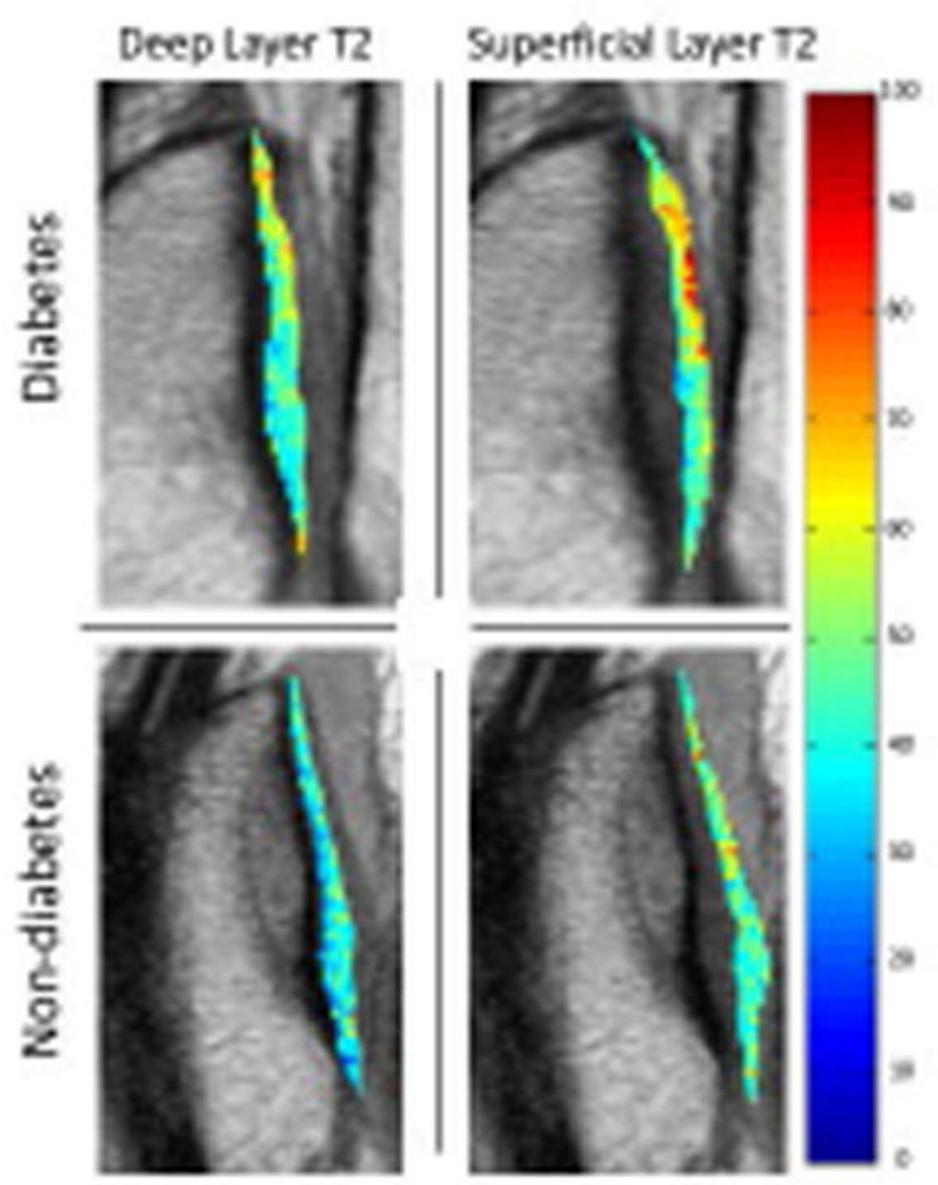

Figure 3.

Sagittal T2 color maps of the patella of the deep and superficial layer of the right knee of a subject with diabetes mellitus and a matched subject without diabetes mellitus. Blue color indicates low, while red color high cartilage T2 values. Cartilage of diabetic subject showed elevated T2 relaxation time (red) compared to the subject without diabetes mellitus, compatible with severer cartilage matrix degeneration of the subject with diabetes mellitus compared to the subjects without diabetes mellitus. 


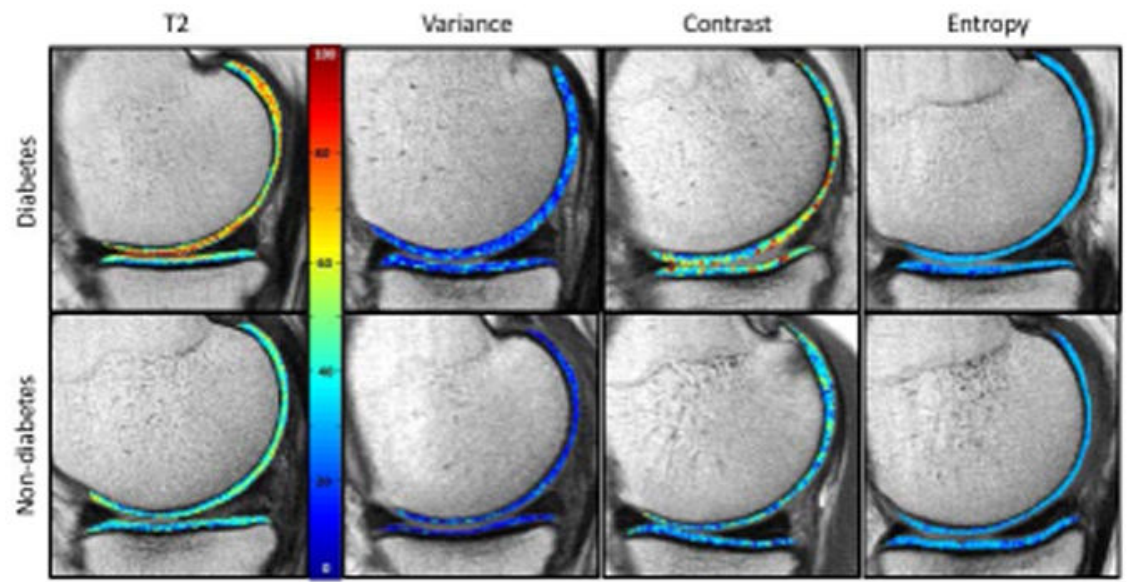

Figure 4.

Sagittal T2 color maps as well as the corresponding texture maps (variance, contrast and entropy) of the medial compartment of the right knee of a subject with diabetes mellitus and a matched subject without diabetes mellitus. On the T2 maps, blue color indicates low, while red color high cartilage T2 values. Cartilage of diabetic subject showed elevated T2 relaxation time compared to the subject without diabetes mellitus. The corresponding texture maps show a wider range of values, displayed through colors, in the subject with compared to the subject without diabetes. Again these findings are compatible with severer cartilage matrix degeneration of the subject with diabetes mellitus compared to the subjects without diabetes mellitus. 


\section{Table 1}

Subject characteristics

\begin{tabular}{|c|c|c|c|}
\hline & Non-diabetics $(\mathrm{n}=208)^{I}$ & Diabetics $(\mathrm{n}=208)^{1}$ & $P$-value \\
\hline \multicolumn{4}{|l|}{ Demographics } \\
\hline Age (years) & $63.27 \pm 9.13$ & $63.00 \pm 8.89$ & $0.76^{2}$ \\
\hline Body mass index $\left(\mathrm{kg} / \mathrm{m}^{2}\right)$ & $31.01 \pm 4.44$ & $31.18 \pm 4.54$ & $0.70^{2}$ \\
\hline Height (m) & $1.68 \pm 0.09$ & $1.69 \pm .09$ & $0.69^{2}$ \\
\hline Females $[\mathrm{n}(\%)]$ & $111(53.4 \%)$ & $111(53.4 \%)$ & $1.00^{3}$ \\
\hline Males [n (\%)] & $97(46.6 \%)$ & $97(46.6 \%)$ & $1.00^{3}$ \\
\hline Physical Activity Score for the Elderly & $154.97 \pm 85.46$ & $146.34 \pm 79.26$ & $0.29^{2}$ \\
\hline Right knee Kellgren-Lawrence & & & $0.93^{3}$ \\
\hline Grade $0[\mathrm{n}(\%)]$ & $86(41.3 \%)$ & $83(39.9 \%)$ & \\
\hline Grade $1[\mathrm{n}(\%)]$ & $49(23.6 \%)$ & $52(25.0 \%)$ & \\
\hline Grade $2[\mathrm{n}(\%)]$ & $73(35.1 \%)$ & $73(35.1 \%)$ & \\
\hline Race & & & $<0.001^{3}$ \\
\hline Caucasian $[\mathrm{n}(\%)]$ & $82.7 \%$ & $59.6 \%$ & \\
\hline African American [n (\%)] & $14.9 \%$ & $35.6 \%$ & \\
\hline Asian $[\mathrm{n}(\%)]$ & $0.5 \%$ & $1.9 \%$ & \\
\hline Other Non-white [n (\%)] & $1.9 \%$ & $2.9 \%$ & \\
\hline \multicolumn{4}{|l|}{ Health status } \\
\hline Heart attack [n (\%)] & $9(4.3 \%)$ & $10(5.0 \%)$ & $0.77^{3}$ \\
\hline $\begin{array}{l}\text { Stroke, cerebrovascular accident, blood clot or bleeding in brain, or transient } \\
\text { ischemic attack [n(\%)] }\end{array}$ & $6(2.9 \%)$ & $11(5.3 \%)$ & $0.21^{3}$ \\
\hline \multicolumn{4}{|l|}{ Osteoarthritis risk factors } \\
\hline History of knee injury [n (\%)] & $75(36.4 \%)$ & $81(39.1 \%)$ & $0.57^{3}$ \\
\hline History of knee surgery [n (\%)] & $32(15.4 \%)$ & $37(17.8 \%)$ & $0.51^{3}$ \\
\hline Family history of knee replacement surgery [n (\%)] & $28(13.5 \%)$ & $20(9.8 \%)$ & $0.23^{3}$ \\
\hline
\end{tabular}

J Magn Reson Imaging. Author manuscript; available in PMC 2019 February 01. 


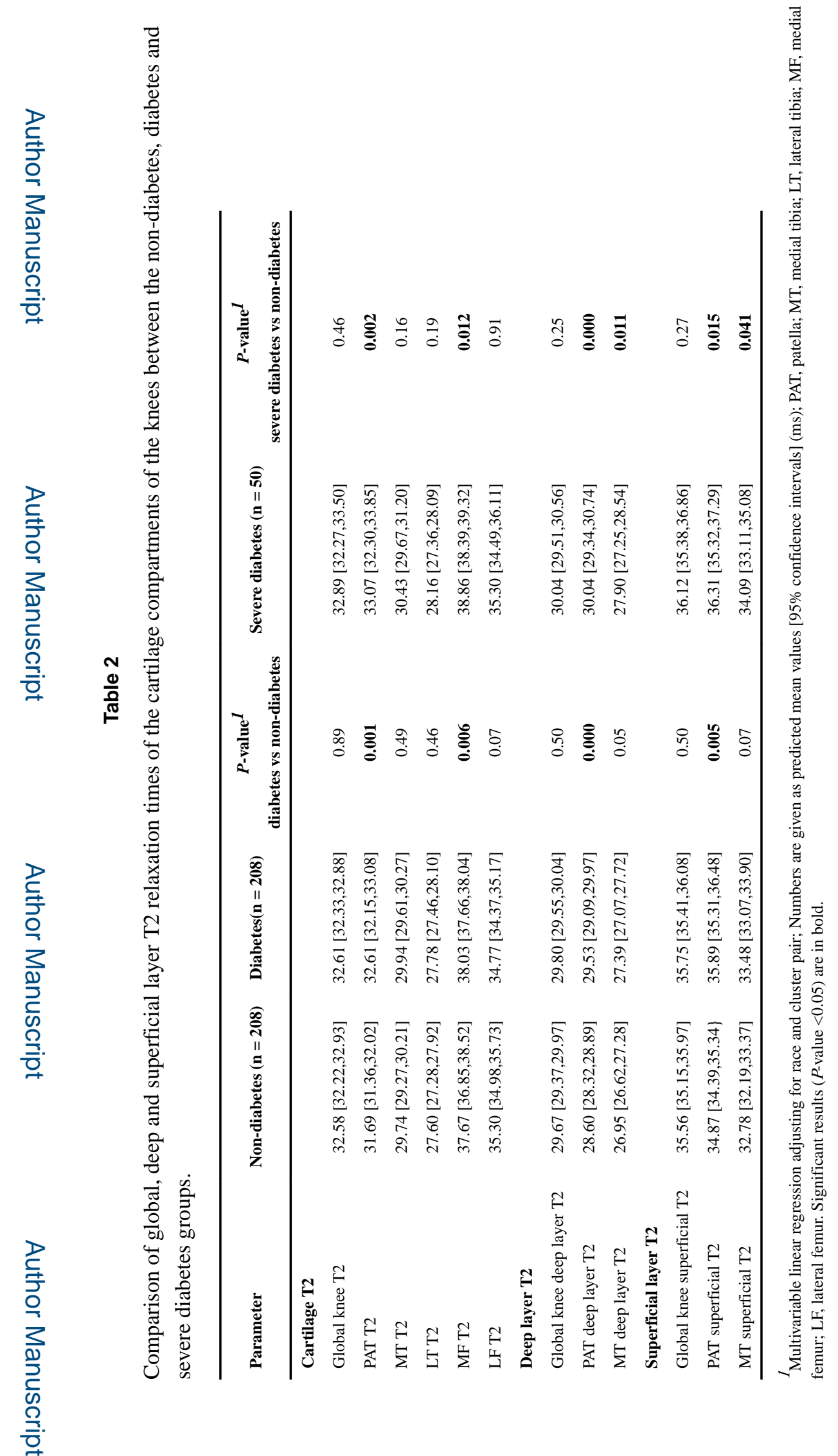

J Magn Reson Imaging. Author manuscript; available in PMC 2019 February 01. 


\section{Table 3}

Comparison of $\mathrm{T} 2$ texture parameters between subjects with and without diabetes mellitus.

\begin{tabular}{lccc}
\hline Parameter & Non-diabetes $(\mathbf{n}=\mathbf{2 0 8})$ & Diabetes $(\mathbf{n}=\mathbf{2 0 8})$ & $\begin{array}{c}\boldsymbol{P}_{\text {-value }} \mathbf{I} \\
\text { diabetes vs. non-diabetes }\end{array}$ \\
\hline Variance & & & \\
Global knee Variance & $213.93[207.42,220.45]$ & $229.35[222.40,236.30]$ & $\mathbf{0 . 0 0 1}$ \\
PAT Variance & $198.68[188.92,208.45]$ & $220.36[210.31,230.42]$ & $\mathbf{0 . 0 0 2}$ \\
MT Variance & $219.69[211.01,228.37]$ & $249.64[238.76,260.51]$ & $\mathbf{0 . 0 0 0}$ \\
Contrast & $296.77[286.87,306.67\}]$ & $316.79[306.17,327.40]$ & $\mathbf{0 . 0 0 2}$ \\
Global knee Contrast & $263.70[250.35,277.05]$ & $291.71[278.16,305.25]$ & $\mathbf{0 . 0 0 2}$ \\
PAT Contrast & $319.60[306.55,332.66]$ & $366.06[348.51,383.61]$ & $\mathbf{0 . 0 0 0}$ \\
MT Contrast & $6.17[6.12,6.21]$ & $6.30[6.27,6.34]$ & \\
Entropy & $5.96[5.90,6.01]$ & $6.09[6.03,6.15]$ & $\mathbf{0 . 0 0 0}$ \\
Global knee Entropy & $5.85[5.79,5.91]$ & $6.04[5.99,6.09]$ & $\mathbf{0 . 0 0 1}$ \\
PAT Entropy & & & $\mathbf{0 . 0 0 0}$ \\
MT Entropy & & & \\
\hline
\end{tabular}

1 Multivariable linear regression adjusting for race and cluster pair; Numbers are given as predicted mean values [95\% confidence intervals] (ms); PAT, patella; MT, medial tibia; Significant result $(P$-values $<0.05)$ are in bold. 


\section{Table 4}

Comparison of WORMS subscores of the knee between subjects with and without diabetes mellitus.

\begin{tabular}{lccc}
\hline WORMS scores & Non-diabetes $(\mathbf{n}=\mathbf{2 0 8})$ & Diabetes $(\mathbf{n}=\mathbf{2 0 8})$ & $\boldsymbol{P}$-value $\boldsymbol{I}$ \\
\hline Cartilage lesions & & & \\
Global knee joint & $4.54[4.02,5.05]$ & $4.11[3.48,4.74]$ & 0.20 \\
PAT & $2.42[2.08,2.75]$ & $2.34[2.01,2.67]$ & 0.68 \\
MT & $0.21[0.10,0.32]$ & $0.16[0.07,0.25]$ & 0.51 \\
LT & $0.67[0.53,0.82]$ & $0.52[0.37,0.68]$ & 0.14 \\
MF & $0.76[0.60,0.92]$ & $0.65[0.49,0.80]$ & 0.32 \\
LF & $0.48[0.33,0.63]$ & $0.45[0.29,0.61]$ & 0.72 \\
Cartilage Max & $2.81[2.52,3.09]$ & $2.57[2.26,2.88]$ & 0.17 \\
Meniscus lesions & & & \\
Bilateral menisci & $1.79[1.48,2.11]$ & $1.84[1.53,2.14]$ & 0.83 \\
Medial meniscus & $0.64[0.50,0.79]$ & $0.85[0.70,0.99]$ & 0.05 \\
Lateral meniscus & $0.61[0.44,0.78]$ & $0.62[0.48,0.76]$ & 0.96 \\
WORMS overall & $7.77[6.94,8.60]$ & $7.12[6.13,8.11]$ & 0.24 \\
\hline
\end{tabular}

1 Multivariable linear regression adjusting for race and cluster pair; Numbers are given as predicted mean values [95\% confidence intervals] (ms); PAT, patella; MT, medial tibia; LT, lateral tibia; MF, medial femur; LF, lateral femur. Significant results $(P$-value $<0.05)$ are in bold. 